\title{
Mass ordering of differential elliptic flow and its violation for $\phi$ mesons
}

\author{
Tetsufumi Hirano, ${ }^{1,}{ }^{*}$ Ulrich Heinz, ${ }^{2,3}$ Dmitri Kharzeev, ${ }^{4}$ Roy Lacey, ${ }^{5}$ and Yasushi Nara ${ }^{6}$ \\ ${ }^{1}$ Department of Physics, The University of Tokyo, Tokyo 113-0033, Japan \\ ${ }^{2}$ Department of Physics, The Ohio State University, Columbus, Ohio 43210, USA \\ ${ }^{3}$ CERN, Physics Department, Theory Division, CH-1211 Geneva 23, Switzerland \\ ${ }^{4}$ Nuclear Theory Group, Physics Department, Brookhaven National Laboratory, Upton, New York 11973-5000, USA \\ ${ }^{5}$ Department of Chemistry, SUNY Stony Brook, Stony Brook, New York 11794-3400, USA \\ ${ }^{6}$ Akita International University, 193-2 Okutsubakidai, Yuwa-Tsubakigawa, Akita 010-1211, Japan
}

(Received 31 October 2007; published 28 April 2008)

\begin{abstract}
We simulate the dynamics of Au+Au collisions at the Relativistic Heavy Ion Collider (RHIC) with a hybrid model that treats the dense early quark-gluon plasma (QGP) stage macroscopically as an ideal fluid but models the dilute late hadron resonance gas (HG) microscopically using a hadronic cascade. By comparing with a pure hydrodynamic approach we identify effects of hadronic viscosity on the transverse momentum spectra and differential elliptic flow $v_{2}\left(p_{T}\right)$. We investigate the dynamical origins of the observed mass ordering of $v_{2}\left(p_{T}\right)$ for identified hadrons, focusing on dissipative effects during the late hadronic stage. Within our approach, we find that, at RHIC energies, much of the finally observed mass splitting is generated during the hadronic stage, due to buildup of additional radial flow. The $\phi$ meson, having a small interaction cross section, does not fully participate in this additional flow. As a result, it violates the mass-ordering pattern for $v_{2}\left(p_{T}\right)$ that is observed for other hadron species. We also show that the early decoupling of the $\phi$ meson from the hadronic rescattering dynamics leads to interesting and unambiguous features in the $p_{T}$ dependence of the nuclear suppression factor $R_{A A}$ and of the $\phi / p$ ratio.
\end{abstract}

DOI: 10.1103/PhysRevC.77.044909

PACS number(s): 25.75.Ld, 12.38.Mh, 24.10.Nz, 25.75.Dw

\section{INTRODUCTION}

A presently hotly debated question is whether the quarkgluon plasma (QGP) created in Au+Au collisions at the Relativistic Heavy Ion Collider (RHIC) [1] represents a "perfect liquid" [2-5], i.e., a fluid whose shear viscosity to entropy ratio $\eta / s$ is at or close to the conjectured [6] lower bound $\frac{\eta}{s}=\frac{1}{4 \pi}$. A key observable in this context is the elliptic flow $v_{2}$ of hadrons emitted anisotropically in noncentral collisions [7]. At the highest RHIC energy of $\sqrt{s}=200 \mathrm{~A} \mathrm{GeV}$, the observed $v_{2}$ values near midrapidity $(|\eta| \lesssim 1)$, for not too large impact parameters $(b \lesssim 7 \mathrm{fm})$ and transverse momenta $\left(p_{T} \lesssim\right.$ $1.5 \mathrm{GeV} / c$ ), agree well with predictions from ideal fluid dynamics [2] (i.e., assuming zero viscosity), including [8,9] the predicted dependence of $v_{2}$ on the transverse momentum $p_{T}$ and hadron masses [10]. Evidence for nonzero shear viscosity in the collision fireball is obtained from deviations from ideal fluid dynamical behavior. This is manifested in the experimental data via a gradual breakdown of the ideal fluid description when collisions are studied at larger impact parameters and at lower energies [11] or when measurements are made away from midrapidity [12-14]. In previous work [15] we have shown that a large (and possibly the dominant) fraction of these deviations from ideal hydrodynamics is due to "late viscosity" caused by dissipative effects in the dilute hadronic rescattering stage that stretches between hadronization of the QGP and final kinetic freeze-out. The question whether there is also non-negligible "early viscosity" in the dense QGP phase remains open. An answer to this question requires a proper

\footnotetext{
*Correspond to hirano@phys.s.u-tokyo.ac.jp
}

viscous hydrodynamic treatment of the QGP fluid, which is presently being pursued vigorously $[4,5,16]$. It also depends on still unknown details of the initial conditions in heavy-ion collisions, in particular the initial spatial eccentricity of the fireball [15,17-19].

In this article we report additional results from the hybrid model study presented in Ref. [15], focusing our attention on a detailed investigation of dissipative effects during the late hadronic rescattering stage. The early QGP stage, including its hadronization, is described by ideal fluid dynamics. Specifically, we address the questions of (i) how radial and elliptic flow evolve during the hadronic stage when it is described by a realistic hadronic rescattering cascade, rather than by an ideal fluid; (ii) how these differences affect the shapes of the finally observed hadronic $p_{T}$ spectra and their differential elliptic flow $v_{2}\left(p_{T}\right)$; and (iii) whether the differences between ideal fluid and realistic kinetic behavior during the late hadronic stage are similar for all hadronic species or whether different magnitudes of their scattering cross sections translate into measurably different characteristics of their observed spectra and elliptic flow.

The article is organized as follows: For completeness, we begin in Sec. II with a short review of the hybrid model [15] employed in this study. Our results are presented in Sec. III, in three subsections organized along the questions raised in the preceding paragraph. We close the article by presenting our conclusions and some perspectives in Sec. IV.

\section{THE MODEL}

Our study is based on a hybrid model that combines an ideal fluid dynamical description of the QGP stage with a realistic 
kinetic simulation of the hadronic stage [15]. Relativistic hydrodynamics is the most relevant formalism to understand the bulk and transport properties of the QGP because it directly connects the collective flow pattern developed during the QGP stage with its equation of state (EOS). It is based on the key assumption of local thermalization. Because this assumption breaks down during both the very anisotropic initial matter formation stage and the dilute late hadronic rescattering stage, the hydrodynamic model can be applied only during the intermediate period, between initial thermalization after about $1 \mathrm{fm} / c$ [2] and the completion of the quark-hadron transition, which, in central $\mathrm{Au}+\mathrm{Au}$ collisions at RHIC energies, happens after about $10 \mathrm{fm} / c$.

In absence of a nonequilibrium dynamical model for the early pre-equilibrium stage of the collision, its output is replaced by a set of initial conditions for the hydrodynamic evolution that are tuned to experimental measurements of the final state in central $(b=0)$ collisions [2]. To describe the breakdown of the hydrodynamical model during the late hadronic stage, due to expansion and dilution of the matter, one has two options: One can either impose a sudden transition from thermalized matter to noninteracting, free-streaming hadrons through the Cooper-Frye prescription [20], imposed at a suitable value of the decoupling temperature $T_{\mathrm{dec}}$ or

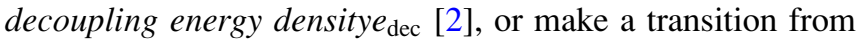
the macroscopic hydrodynamic description to a microscopic kinetic description at a suitable value for the switching temperature $T_{\mathrm{sw}}$ where both descriptions are simultaneously valid [15,21-24], letting the subsequent kinetic decoupling play itself out automatically by following the microscopic evolution until all interactions have ceased. We here use both approaches alternatively to isolate effects that are specifically caused by dissipative effects in the hadron rescattering cascade.

\section{A. Ideal hydrodynamics}

For the space-time evolution of the perfect QGP fluid we solve numerically the equations of motion of ideal fluid dynamics, for a given initial state, in three spatial dimensions and in time $[(3+1)-d$ ideal hydrodynamics [13]:

$$
\begin{aligned}
\partial_{\mu} T^{\mu \nu} & =0, \\
T^{\mu \nu} & =(e+p) u^{\mu} u^{\nu}-p g^{\mu \nu} .
\end{aligned}
$$

Here $e, p$, and $u^{\mu}$ are energy density, pressure, and fourvelocity of the fluid, respectively. Due to its smallness at collider energies, we neglect the net baryon density. As an algorithm to solve the above equations we choose the piecewise parabolic method (PPM) [25]. It is known to be a very robust scheme for solving nonrelativistic gas dynamics including shock wave formation and has been employed in many fields. We first applied it in [26] to solve Eulerian hydrodynamics for relativistic heavy-ion collisions, Eqs. (1) and (2). Use of this algorithm enables us to describe the space-time evolution of relativistic fluids accurately even if the matter passes through a first-order phase transition. The PPM is a higher-order extension of the piecewise linear method employed, for example, in the relativistic Harten-
Lax-van Leer-Einfeldt (rHLLE) algorithm [27]. We solve Eq. (1) in $\left(\tau, x, y, \eta_{s}\right)$ coordinates [13] where $\left.\tau=\sqrt{t^{2}-z^{2}}\right)$ and $\eta_{s}=\frac{1}{2} \ln [(t+z) /(t-z)]$ are longitudinal proper time and space-time rapidity, respectively, adequate for the description of collisions at ultrarelativistic energies. The grid sizes are $\Delta \tau=0.3 \mathrm{fm} / c, \Delta x=\Delta y=0.3 \mathrm{fm}$, and $\Delta \eta_{s}=0.3$. We have checked the grid size dependence of our final results and observed sufficient convergence with the given choice of grid parameters, as long as smooth initial conditions such as those discussed below are used.

\section{B. Equation of state}

For the high-temperature $\left(T>T_{c}=170 \mathrm{MeV}\right)$ QGP phase we use the EOS of massless noninteracting parton gas $(u, d, s$ quarks and gluons) with a bag pressure $B$ :

$$
p=\frac{1}{3}(e-4 B) \text {. }
$$

The bag constant is tuned to $B^{\frac{1}{4}}=247.19 \mathrm{MeV}$ to ensure a first-order phase transition to a hadron resonance gas at critical temperature $T_{c}=170 \mathrm{MeV}$. The hadron resonance gas model at $T<T_{c}$ includes all hadrons up to the mass of the $\Delta(1232)$ resonance. Systematic studies with various models of the EOS, including a more realistic cross-over one, will be discussed elsewhere.

For a meaningful discrimination between the ideal fluid and hadron cascade descriptions of the hadron phase, and a realistic direct comparison of hydrodynamic results with experimental data, our hadron resonance gas EOS implements chemical freeze-out at $T_{\text {chem }}=T_{c}=170 \mathrm{MeV}$, as observed in RHIC collisions [28]. This is achieved by introducing appropriate temperature-dependent chemical potentials $\mu_{i}(T)$ for all hadronic species $i$ in such a way that their numbers $\tilde{N}_{i}$, including all decay contributions from higher-lying resonances, $\tilde{N}_{i}=N_{i}+\sum_{R} b_{R \rightarrow i X} N_{R}$, are conserved during the evolution [14,29-33]. [Here $N_{i}$ is the number of the $i$ th hadron, and $b_{R \rightarrow i X}$ is the effective branching ratio (a product of branching ratio and degeneracy) of a decay process $R \rightarrow$ $i+X$.] In this "PCE model" [14] only strongly interacting resonances with large decay widths (whose decays do not alter $\tilde{N}_{i}$ ) remain chemically equilibrated below the chemical freeze-out temperature.

The hadronic chemical composition described by hydrodynamics using the PCE model EOS is roughly consistent with that of the hadronic cascade models, as long as the latter are initialized at $T_{\mathrm{sw}}$ with thermal and chemical equilibrium distributions [34]. This is crucial for a meaningful comparison between hydrodynamic and kinetic descriptions of hadronic matter because the chemical composition of the hadron resonance gas has a significant influence on the hydrodynamic evolution of the hadronic transverse momentum spectra [3]: although the nonequilibrium hadronic chemical potentials $\mu_{i}(T)$ do not affect the EOS $p(e)$ of the hadronic phase [14], and thus lead to almost identical evolution of radial flow and total momentum anisotropy as for a chemically equilibrated hadron gas, they significantly alter the relationship between energy density and temperature, leading to cooler temperatures and hence to steeper transverse momentum spectra at identical 
kinetic decoupling energy densities [14]. This effect is seen most dramatically in the time dependence of the mean transverse momentum for pions [3]: $\left\langle p_{T}\right\rangle_{\pi}$ decreases with proper time after chemical freeze-out, whereas with continued hadronic chemical equilibrium it would increase with time. Clear conclusions about hadronic dissipative effects on the shapes of the transverse momentum spectra can therefore be drawn only from a comparison with hydrodynamic models that implement chemical and kinetic freeze-out separately.

\section{Initial conditions}

Contrary to Ref. [15] where we studied both Glauber model and color glass condensate (CGC) type initial conditions, for the comparative study presented here we concentrate on the Glauber model, suitably generalized to account for the longitudinal structure of particle multiplicity [15,36]. We assume an initial entropy distribution of massless partons according to

$$
\begin{aligned}
\frac{d S}{d \eta_{s} d^{2} x_{\perp}}= & \frac{C}{1+\alpha} \theta\left(Y_{b}-\left|\eta_{s}\right|\right) f^{p p}\left(\eta_{s}\right) \\
& \times\left[\alpha\left(\frac{Y_{b}-\eta_{s}}{Y_{b}} \frac{d N_{\mathrm{part}}^{A}}{d^{2} x_{\perp}}+\frac{Y_{b}+\eta_{s}}{Y_{b}} \frac{d N_{\mathrm{part}}^{B}}{d^{2} x_{\perp}}\right)\right. \\
& \left.+(1-\alpha) \frac{d N_{\mathrm{coll}}}{d^{2} x_{\perp}}\right],
\end{aligned}
$$

where $\boldsymbol{x}_{\perp}=(x, y)$ is the position perpendicular to the beam axis, $C$ is a normalization factor, the "soft fraction" $\alpha$ is explained below, the parameter $Y_{b}$ is the beam rapidity, and $f^{p p}$ is a suitable parametrization of the shape of rapidity distribution in $p p$ collisions:

$$
f^{p p}\left(\eta_{s}\right)=\exp \left[-\theta\left(\left|\eta_{s}\right|-\Delta \eta\right) \frac{\left(\left|\eta_{s}\right|-\Delta \eta\right)^{2}}{\sigma_{\eta}^{2}}\right] .
$$

We study $\mathrm{Au}+\mathrm{Au}$ collisions at $\sqrt{s}=200 \mathrm{~A} \mathrm{GeV}$ and use $C=$ $24, \Delta \eta=1.3$, and $\sigma_{\eta}=2.1$, so chosen as to reproduce the charged hadron pseudorapidity distributions measured in these collisions [37]. $N_{\text {part }}^{A, B}$ and $N_{\text {coll }}$ are the number of wounded nucleons in each of the two nuclei and the number of binary nucleon-nucleon collisions, respectively. These are calculated from the Glauber model nuclear thickness function $T_{A, B}\left(x_{\perp}\right)$ [38]:

$$
\begin{aligned}
& \frac{d N_{\text {part }}^{A}}{d^{2} x_{\perp}}=T_{A}\left(r_{+}\right)\left[1-\left(1-\frac{\sigma_{N N}^{\text {in }} T_{B}\left(r_{-}\right)}{B}\right)^{B}\right], \\
& \frac{d N_{\text {part }}^{B}}{d^{2} x_{\perp}}=T_{B}\left(r_{-}\right)\left[1-\left(1-\frac{\sigma_{N N}^{\text {in }} T_{A}\left(r_{+}\right)}{A}\right)^{A}\right], \\
& \frac{d N_{\text {coll }}}{d^{2} x_{\perp}}=\sigma_{N N}^{\text {in }} T_{A}\left(r_{+}\right) T_{B}\left(r_{-}\right) .
\end{aligned}
$$

Here $\sigma_{N N}^{\text {in }}=42 \mathrm{mb}$ is the inelastic nucleon-nucleon cross section, and $r_{ \pm}=\left[\left(x \pm \frac{1}{2} b\right)^{2}+y^{2}\right]^{1 / 2}$ (where $b$ is the impact parameter).

The soft/hard fraction $\alpha=0.85$ was adjusted to reproduce the measured centrality dependence [39] of the charged hadron multiplicity at midrapidity. At $\eta_{s}=0$, Eq. (4) reduces to $\frac{d S}{d \eta_{s} d^{2} x_{\perp}} \propto \frac{1}{1+\alpha}\left[\alpha\left(n_{\text {part }}^{A}+n_{\text {part }}^{B}\right)+(1-\alpha) n_{\text {coll }}\right]$, where $n \equiv \frac{d N}{d^{2} x_{\perp}}$ [40]; this parametrization is equivalent to the one used in Ref. [41], $\propto\left[\frac{1-x}{2}\left(n_{\text {part }}^{A}+n_{\text {part }}^{B}\right)+x n_{\text {coll }}\right]$, with $x=\frac{1-\alpha}{1+\alpha}$. From Eq. (4), we can compute the entropy density at the initial time $\tau_{0}=0.6 \mathrm{fm} / c$ [2] of the hydrodynamic evolution, $s\left(\tau_{0}, \boldsymbol{x}_{\perp}, \eta_{s}\right)=\frac{d S}{\tau_{0} d \eta_{s} d^{2} x_{\perp}}$, which provides the initial energy density and pressure distributions through the tabulated EOS described above.

Glauber model initial conditions have a long tradition for hydrodynamic simulations of heavy-ion collisions. In our previous study [15] we showed that with such initial conditions "late viscosity" effects during the dilute hadronic rescattering stage are sufficient to explain all observed deviations of elliptic flow measurements from ideal fluid dynamical predictions. No significant additional viscous effects during the early QGP stage were necessary. We also noted, however, that this conclusion depends crucially on this particular choice of initial conditions, specifically the initial source eccentricity predicted by the Glauber model. The good agreement between theory and experiment disappears when one instead calculates the initial conditions from the KLN model [17-19,41-43], which is based on CGC ideas and, for the same impact parameter, produces almost $30 \%$ larger source eccentricities. If nature gives preference to such more eccentric initial conditions, additional viscous effects and/or a softer EOS for the QGP stage may be needed to reproduce the experimental data $[15,44]$. Here, we will not pursue this line of thought any further but focus on the case of Glauber model initial conditions and the specific modifications of hadron spectra and flow caused by "late hadronic viscosity."

\section{Hadronic cascade model}

In our hybrid model simulations we switch from ideal hydrodynamics to a hadronic cascade model at the switching temperature $T_{\mathrm{sw}}=169 \mathrm{MeV}$. The subsequent hadronic rescattering cascade is modeled by JAM [45], initialized with hadrons distributed according to the hydrodynamic model output, calculated with the Cooper-Frye formula [20] along the $T_{\mathrm{sw}}=169 \mathrm{MeV}$ hypersurface rejecting inward-going particles. We have checked [15] that switching from an ideal hydrodynamic to a hybrid model description does not entail a major readjustment of initial conditions: Keeping the same initial conditions and hard/soft fraction $\alpha$ as previously determined within a purely hydrodynamic approach (see Refs. [2,14] for a detailed discussion of that procedure) we find [15] that the centrality dependence of $d N_{\mathrm{ch}} / d \eta$ at midrapidity remains consistent with the experimental data even if we switch below $T_{\mathrm{sw}}$ to the hadronic cascade. Effects on the hadron spectra and elliptic flow are significant, however, and will be discussed in the next section.

As customary in hadronic cascade models [45-47], JAM implements experimental hadronic scattering cross section data where available and uses the additive quark model where data do not exist, assuming the following formula for the total 
cross section:

$$
\sigma_{\mathrm{tot}}=\sigma_{N N}^{\mathrm{tot}} \frac{n_{1}}{3} \frac{n_{2}}{3}\left(1-0.4 \frac{n_{s 1}}{n_{1}}\right)\left(1-0.4 \frac{n_{s 2}}{n_{2}}\right) .
$$

Here $\sigma_{N N}^{\text {tot }}$ is the total nucleon-nucleon cross section, $n_{i}$ is the number of constituent quarks in a hadron, and $n_{s i}$ is the number of strange quarks in a hadron. For hadrons composed entirely of strange quarks, such as $\phi=(s \bar{s})$ and $\Omega=(s s s)$, the cross sections become very small, due to the suppression factors in brackets in Eq. (9). We calculate spectra for $\phi$ mesons in Sec. III C; the decay channels for $\phi$ mesons are switched off in the hadronic cascade calculations. Because the life time of $\phi$ mesons $(\approx 46 \mathrm{fm} / c)$ is longer than the typical life time of the system $(\sim 10-20 \mathrm{fm} / c)$, and the number of $\phi$ mesons is small compared to pions, kaons, and nucleons, this prescription is not expected to affect the bulk space-time evolution during the hadronic stage. We stress that the prediction of a violation of mass scaling of the differential elliptic flow $v_{2}\left(p_{T}\right)$ made in this article crucially depends on the reduced hadronic rescattering cross section for $\phi$ mesons and thus presents an important test of this widely accepted assumption.

\section{RESULTS}

In Ref. [15] we investigated the effect of hadronic dissipation on elliptic flow and found that it significantly suppresses the $p_{T}$ integrated $v_{2}$ at forward and backward rapidity and in peripheral collisions. In the following we explore the origins of this finding in more detail, by investigating hadronic dissipative effects on hadron spectra and differential elliptic flow $v_{2}\left(p_{T}\right)$. We finally explore specifically the spectra and elliptic flow of $\phi$ mesons as an example of a hadron that is only weakly coupled to the rest of the expanding hadronic fireball.

\section{A. Hadronic dissipative effects on spectra and elliptic flow}

In this subsection, we compare results from the hybrid model with the ones from ideal hydrodynamics. In ideal hydrodynamic calculations it is assumed that even the late hadron resonance gas phase is characterized by essentially vanishing mean free paths and thus behaves as a perfect fluid, all the way down to kinetic decoupling of the hadron momenta at $T_{\text {th }}=100 \mathrm{MeV}$. (This value is obtained by a simultaneous fit of the pion and proton spectra in central collisions that allows us to separate the effects of radial flow and thermal motion at kinetic freeze-out [2].) As discussed, chemical freeze-out is implemented at $T_{\text {chem }}=170 \mathrm{MeV}$ by using an EOS with nonequilibrium chemical potentials that hold the stable particle yields constant (and close to the ones in the cascade model approach) during the hydrodynamic evolution of the hadronic phase. The key difference between the hydrodynamic and hybrid model approaches is, thus, the finite mean free path for momentum-changing collisions in the hadronic cascade.

In Fig. 1, $p_{T}$ spectra for protons and pions are shown for both, the hybrid model and the ideal hydrodynamic approach. For comparison, we also plot the $p_{T}$ spectra without hadronic rescattering, obtained by setting all cross sections to zero
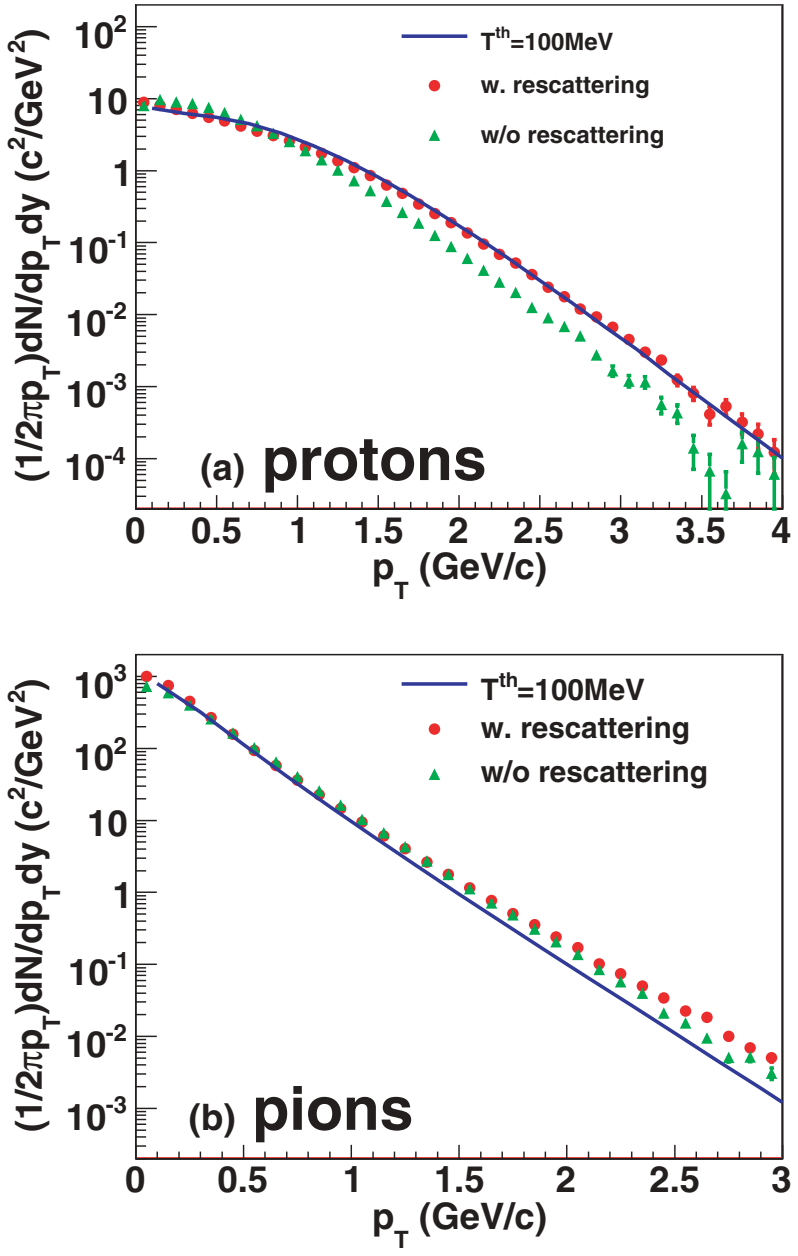

FIG. 1. (Color online) $p_{T}$ spectra with and without hadronic rescattering for (a) protons and (b) pions at midrapidity for $\mathrm{Au}+\mathrm{Au}$ collisions at $b=2 \mathrm{fm}$, compared with results from ideal hydrodynamics decoupling at $T_{\text {th }}=100 \mathrm{MeV}$.

in the hadron cascade or by setting $T_{\mathrm{th}}=T_{\mathrm{sw}}=169 \mathrm{MeV}$ in the hydrodynamic approach (both procedures give the same spectra, by construction). Note here that we include contributions from all resonances (except for weak decays unless explicitly noted otherwise) in ideal hydrodynamic and hybrid-model results. One sees that hadronic rescattering in the JAM cascade pushes the protons to higher $p_{T}$ in exactly the same way as the growing radial flow does in the hydrodynamic approach, if one chooses for the latter a kinetic decoupling temperature of $T_{\mathrm{th}}=100 \mathrm{MeV}$. The reasonable fit of the measured proton $p_{T}$ spectra [48] up to $p_{T} \sim 1.5 \mathrm{GeV} / c$ by the hydrodynamic model $[2,14,32]$ thus persists in the hybrid model approach (see Fig. 3 in the following subsection).

The lack of visible dissipative effects on the proton spectra is probably an artifact caused by a judicial choice of the kinetic freeze-out temperature $T_{\text {th }}=100 \mathrm{MeV}$ in the hydrodynamic approach, which was driven by the wish to reproduce the measured proton spectra with this model. This accident does not repeat itself for the pions, shown in Fig. 1(b). For pions, the $p_{T}$ spectrum becomes slightly steeper when evolved hydrodynamically (the steepening effects due to cooling are 
not quite compensated by the increasing radial flow), whereas it gets hardened by hadronic rescattering effects in the hybrid approach.

This pattern is consistent with theoretical expectations: In the ideal fluid approach, $p d V$ work in the longitudinal direction reduces the transverse energy per unit rapidity $[49,50]$. Because pions dominate the medium but their number is fixed after chemical freeze-out, this leads to a decrease of the average $p_{T}$ per pion [3], explaining the steeper pion spectrum from ideal hydrodynamics. (This argument is not quantitative because it neglects the shifting balance of transverse energy carried by pions and heavier particles such as protons which are more strongly affected by the developing radial flow [3]. Also note that it does not remain true if a chemical equilibrium EOS is used in the hadronic phase where the pion number decreases with temperature and the average transverse energy per pion thus increases [3].) In contrast to the ideal fluid, the hadron gas in the JAM cascade is highly viscous. Shear viscosity is known to reduce the longitudinal and increase the transverse pressure [16], reducing the loss of transverse energy due to longitudinal $p d V$ work and increasing the transverse flow due to larger transverse pressure gradients [16]. In addition, there are viscous corrections to the (flow-boosted) thermal equilibrium form of the distribution function at kinetic freeze-out that lead to an additional viscous distortion of the $p_{T}$ spectrum, which actually increases with $p_{T}^{2}$ [51]. For Bjorken expansion of a homogeneous cylinder this distortion can be written analytically as [51]

$$
\frac{d N}{p_{T} d p_{T}} \approx\left(1+\frac{\Gamma_{s}}{4 \tau_{f} T^{2}} p_{T}^{2}\right) \frac{d N_{0}}{p_{T} d p_{T}},
$$

where $\frac{d N_{0}}{p_{T} d p_{T}}$ is the spectrum calculated from a boosted thermal equilibrium distribution along the decoupling surface at freeze-out time $\tau_{f}$ and temperature $T$, and the expression in brackets preceding it is the $p_{T}^{2}$-dependent viscous correction, parametrized by the sound attenuation length $\Gamma_{s}=\frac{4}{3} \frac{\eta}{s T}$ (where $\eta$ is the shear viscosity).

The viscous flattening of the pion spectrum relative to the pure hydrodynamic approach seen in Fig. 1(b) receives contributions from both factors in Eq. (10): $\frac{d N_{0}}{p_{T} d p_{T}}$ is flattened by the larger transverse flow generated by the viscously increased transverse pressure, and additional flattening comes from the factor in brackets, due to a nonzero value for $\Gamma_{s}$ in a viscous fluid. We do not know which of the two effects is larger; we note only that the pion spectrum from the hybrid model can be fitted very well by simply multiplying the hydrodynamic model spectrum with the factor in brackets in Eq. (10), taking $T=T_{\text {th }}=100 \mathrm{MeV}$ and adjusting $\Gamma_{s} / \tau_{f}=$ 0.01 . How meaningful such a fit is [given that the form (10) makes unrealistic assumptions about the fireball expansion] remains to be seen when realistic viscous hydrodynamic studies become available.

Although these considerations provide a qualitative explanation for the harder pion $p_{T}$-spectrum from the JAM cascade compared to ideal hydrodynamics, the same arguments should also hold for protons where no such effects are seen in Fig. 1(a). As already stated, this is presumably a consequence of an accidental cancellation of delicate thermal and flow

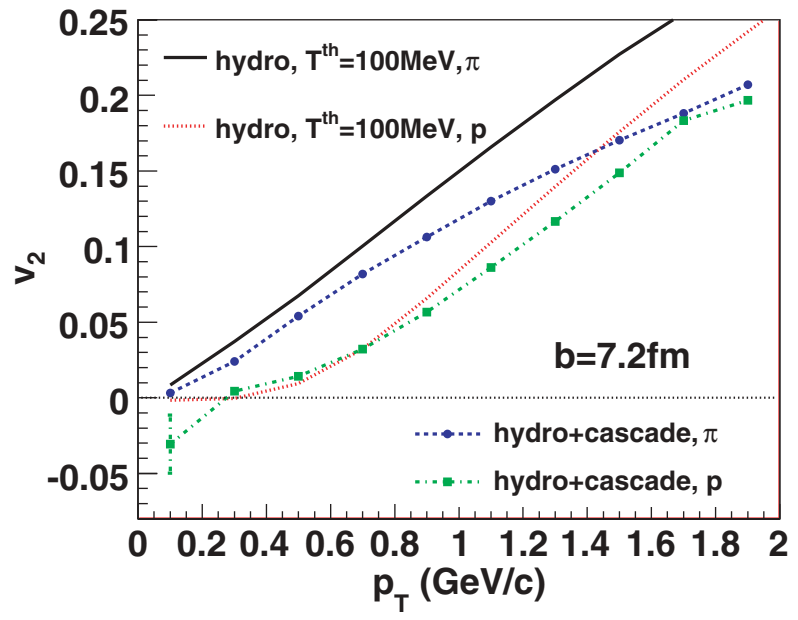

FIG. 2. (Color online) $v_{2}\left(p_{T}\right)$ for pions and protons in $|\eta|<1.3$ at $b=7.2 \mathrm{fm}$. Results for pions (solid) and protons (dotted) from ideal hydrodynamics with $T_{\text {th }}=100 \mathrm{MeV}$ are compared with the ones for pions (dashed) and protons (dash-dotted) from the hybrid model.

effects with viscous corrections for our specific choice of $T_{\text {th }}$ in the hydrodynamic model. Again, a full understanding of these results may require comparison with a viscous hydrodynamic treatment $[4,5]$.

Figure 2 shows the $p_{T}$ dependence of $v_{2}$ for pions and protons in semicentral $\mathrm{Au}+\mathrm{Au}$ collisions $(b=7.2 \mathrm{fm})$ at midrapidity $(|\eta|<1.3)$, comparing results from the hybrid model with ideal hydrodynamics. Whereas, after an initial quadratic rise that extends over a larger $p_{T}$ range for the heavier protons than the lighter pions [10], the differential elliptic flow $v_{2}\left(p_{T}\right)$ from ideal hydrodynamics increases almost linearly with $p_{T}$, this increase is tempered in the results from the hadronic cascade. The differences between the two models is seen to grow with increasing $p_{T}$. Again, this is qualitatively just as expected from shear viscous effects [4,5,51]. Obviously, the different transport properties of the hadronic matter in JAM and in hydrodynamics are seen more clearly in the differential elliptic flow $v_{2}\left(p_{T}\right)$ than in the $p_{T}$ spectra.

\section{B. Spectra and elliptic flow for $\pi, K$, and $p$}

In this subsection, we compare our results from the hybrid model with experimental data for identified hadrons. In Fig. 3, transverse-momentum spectra for pions, kaons, and protons from the hybrid model are compared with PHENIX data [48] for three impact parameters (centrality classes) as shown in the figure. (The impact parameters are adjusted to give the correct average number of participants for each centrality class, as quoted in Ref. [48].) In all cases, the experimental data are reasonably well reproduced by the hybrid model for low transverse momenta to $p_{T} \sim 1.5-2.0 \mathrm{GeV} / c$. Additional components (such as thermal quark recombination and jet fragmentation, including energy loss of fast partons in the fireball medium) would be required to reproduce the data above $p_{T} \sim 1.5 \mathrm{GeV} / c$. It should be emphasized that, unlike in the purely hydrodynamic approach where the $p_{T}$ slope is 

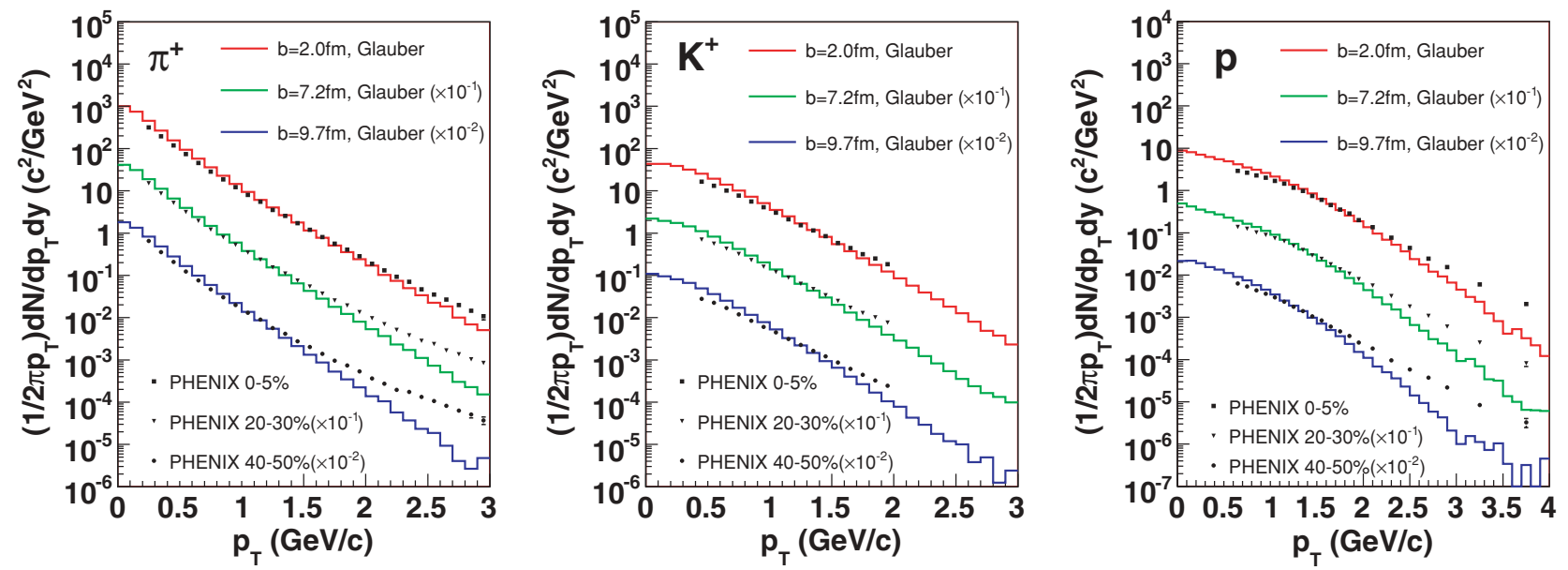

FIG. 3. (Color online) Centrality dependences of the $p_{T}$ spectra for (a) pions, (b) kaons, and (c) protons obtained from our hydro+cascade hybrid model, compared with data from the PHENIX Collaboration [48] for $200 \mathrm{~A} \mathrm{GeV} \mathrm{Au+Au} \mathrm{collisions.} \mathrm{Impact} \mathrm{parameters} \mathrm{are} \mathrm{(from} \mathrm{top}$ to bottom) $b=2.0,7.2$, and $9.7 \mathrm{fm}$, corresponding to the $0-5 \%, 20-30 \%$, and $30-40 \%$ centrality ranges, respectively.

controlled by the choice of kinetic freeze-out temperature and the correct hadron yields are ensured by appropriate choice of nonequilibrium hadron chemical potentials (see Sec. II B), the hybrid model has no adjustable parameters to reproduce both slope and normalization of the transverse-momentum spectra. Hadronic cascade processes automatically describe both chemical and kinetic freeze-out.

In Figure 4, we compare the $p_{T}$ dependence of $v_{2}$ for pions, kaons, and protons with the STAR data for $v_{2}\{2\}$ [52] for four centrality classes. For the $0-5 \%$ centrality class we show only pions because the quality of the kaon and proton data at this centrality is insufficient for a meaningful comparison with theory. The hybrid model correctly describes the mass ordering of the differential elliptic flow, $v_{2}^{\pi}\left(p_{T}\right)>v_{2}^{K}\left(p_{T}\right)>v_{2}^{p}\left(p_{T}\right)$, as seen in the data within the low- $p_{T}$ region covered by the figure. Quantitatively, it provides a reasonable description up to $50 \%$ centrality, except for the most central collisions: Our result for pions at $b=2.0 \mathrm{fm}$ is significantly smaller than the data. This can be attributed to the absence of eccentricity fluctuations in our model calculations [19,53].

To better understand the origin of the mass ordering in $v_{2}\left(p_{T}\right)$, we compare in Fig. 5, for a selected impact parameter of $b=7.2 \mathrm{fm}$, the above hybrid model result with a calculation where all hadronic rescattering is turned off, allowing only for decay of the unstable hadron resonances. Whereas just after hadronization the differential elliptic flow $v_{2}\left(p_{T}\right)$ for pions and protons looks very similar, the mass splitting gets strongly enhanced by hadronic rescattering. The smallness of the pionproton mass splitting at $T_{\mathrm{sw}}$ is partially accidental, because the splitting caused by the radial flow already established during the hydrodynamic QGP phase [10] is significantly decreased by the effect of resonance decays that reduces the pion elliptic flow $v_{2}^{\pi}\left(p_{T}\right)$ by about $15 \%$ [26,54]. Hadronic evolution below $T_{\mathrm{sw}}$ steepens the slope of $v_{2}\left(p_{T}\right)$ for pions [14], due to the generation of additional (integrated) $v_{2}$ and the reduction of their mean transverse momentum $\left\langle p_{T}\right\rangle_{\pi}$ [3]. [Note that for pions the slope of $v_{2}\left(p_{T}\right)$ can be simply approximated as $\left.d v_{2}\left(p_{T}\right) / d p_{T} \approx v_{2} /\left\langle p_{T}\right\rangle[3].\right]$

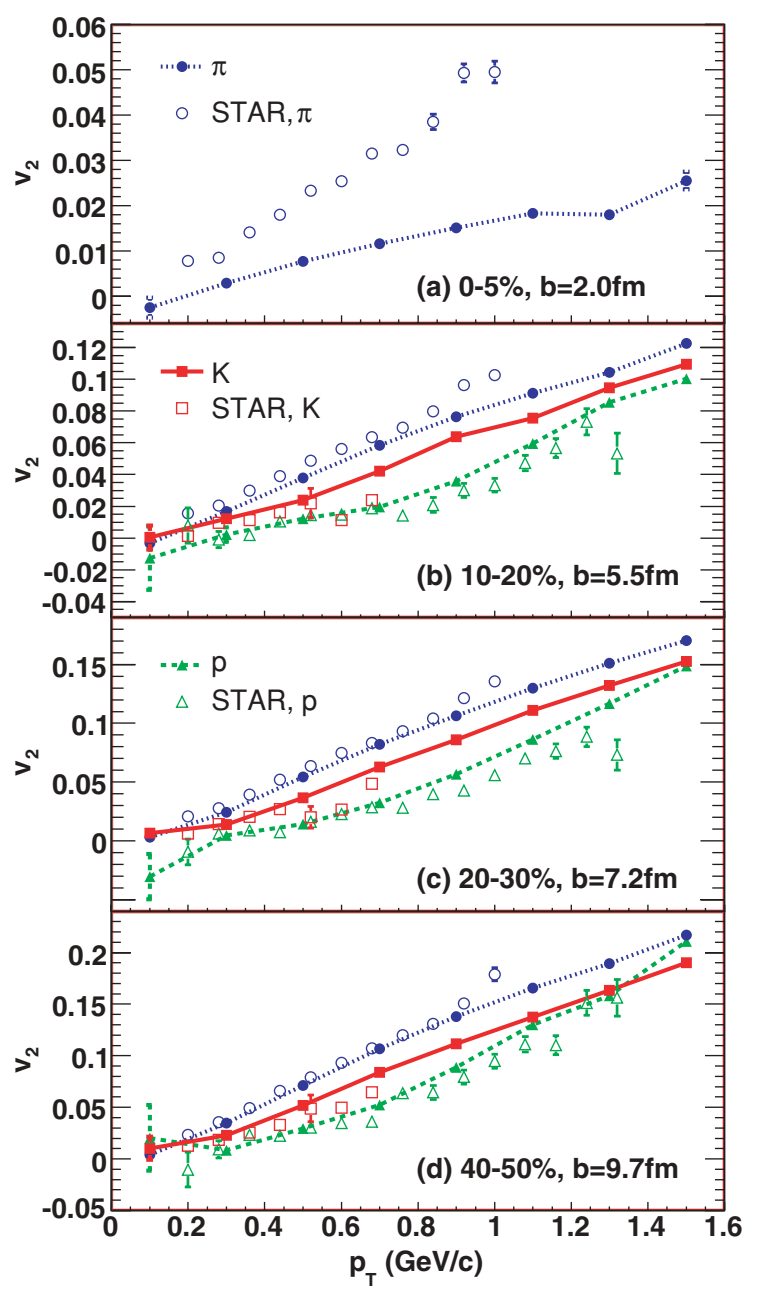

FIG. 4. (Color online) Transverse-momentum dependence of the elliptic flow coefficient $v_{2}$ for pions (dotted blue), kaons (solid red), and protons (dashed green) from the hybrid model, compared with STAR data for $v_{2}\{2\}$ from $200 \mathrm{~A} \mathrm{GeV} \mathrm{Au+Au} \mathrm{collisions,} \mathrm{in} \mathrm{four}$ centrality classes [52]. 
For heavy hadrons, however, radial flow reduces $v_{2}$ at low $p_{T}$ [10]. Assuming positive elliptic flow, $v_{\perp}(\varphi=0, \pi)>$ $v_{\perp}\left(\varphi=\frac{\pi}{2}, \frac{3 \pi}{2}\right)$, the stronger transverse flow $v_{\perp}$ in the reaction plane pushes heavy particles to larger $p_{T}$ more efficiently in the reaction plane than perpendicular to it. In extreme cases [10] this can, for heavy particles, even lead to a depletion of low- $p_{T}$ emission into the reaction plane when compared with out-of-plane emission, i.e., to a negative $v_{2}\left(p_{T}\right)$ at low $p_{T}$ (even though their $p_{T}$-integrated total elliptic flow $v_{2}$ is positive). But even without going to extremes, this mechanism generically reduces $v_{2}\left(p_{T}\right)$ at low $p_{T}$ for heavy hadrons. So it is the generation of additional radial flow in the hadronic stage that is responsible for (most of) the mass splitting of $v_{2}\left(p_{T}\right)$ observed in the low- $p_{T}$ region.

This mechanism works even if the (extra) radial flow is not perfectly hydrodynamic, i.e., if (as is the case in the hadron cascade) the system does not remain fully thermalized, with locally isotropic momentum distributions. Any type of anisotropic collective transverse motion will cause such a mass splitting of $v_{2}\left(p_{T}\right)$ at low $p_{T}$, as long as the hadron in question participates in the flow. It is worth mentioning that in hydrodynamic calculations about half of the final radial flow in $\mathrm{Au}+\mathrm{Au}$ collisions at RHIC is generated during the hadronic stage (see Fig. 7 in Ref. [55] and Fig. 5 in Ref. [14]). A similar increase in radial flow generated by the JAM cascade is documented in Fig. 1(a).

From these observations we conclude that the large magnitude of the integrated $v_{2}$ and the strong mass ordering of the differential $v_{2}\left(p_{T}\right)$ observed at RHIC result from a subtle interplay between perfect fluid dynamics of the early QGP stage and dissipative dynamics of the late hadronic stage: The large magnitude of $v_{2}$ is due to the large overall momentum anisotropy, generated predominantly in the early QGP stage, whereas the strong mass splitting between the slopes of $v_{2}\left(p_{T}\right)$ at low $p_{T}$ reflects the redistribution of this momentum anisotropy among the different hadron species, driven by the continuing radial acceleration and cooling of the matter during the hadronic rescattering phase.

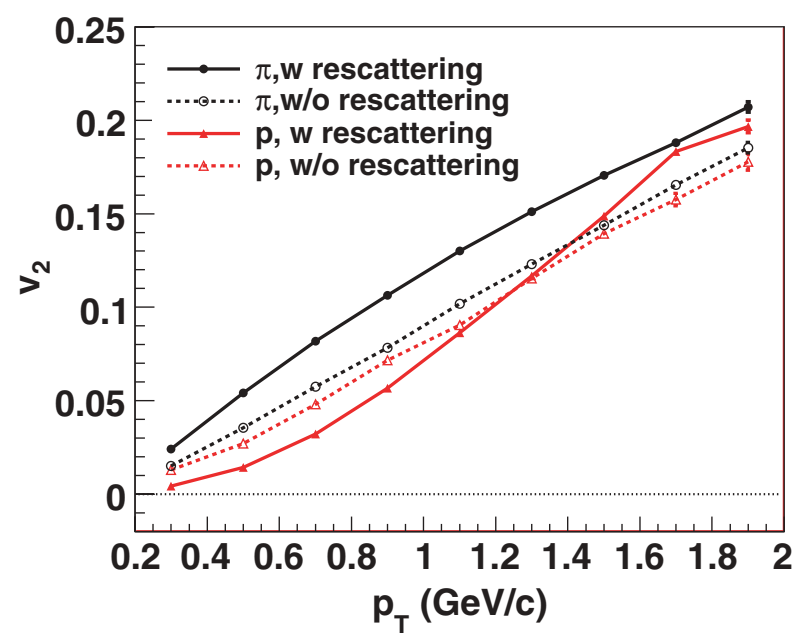

FIG. 5. (Color online) Transverse-momentum dependence of the elliptic flow parameter for pions and protons. Solid (dashed) lines are with (without) hadronic rescattering.

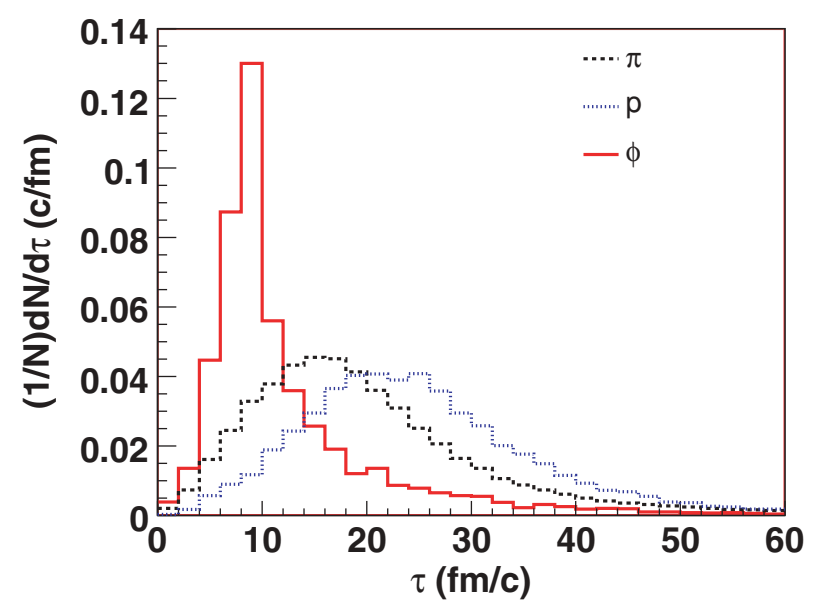

FIG. 6. (Color online) Normalized distribution of freeze-out times for pions (dashed), protons (dotted), and $\phi$ mesons (solid) for $|y|<1$ in $\mathrm{Au}+\mathrm{Au}$ collisions at $b=2.0 \mathrm{fm}$.

\section{Spectra and elliptic flow for $\phi$ mesons}

As noted in Sec. IID, $\phi$ mesons (consisting of strange quarks) have considerably smaller scattering cross sections in JAM than nonstrange hadrons [56]. They are therefore expected to show larger dissipative effects in our hybrid model and to not fully participate in the additional radial flow generated during the hadronic rescattering stage. In kinetic theory language, one expects that the $\phi$ mesons decouple from rest of the system earlier than other, nonstrange hadrons [57], thereby possibly opening a window to extract direct information on collective phenomena in the partonic stage from $\phi$-meson spectra [56].

To study $\phi$ mesons in our hybrid model we stabilize them by turning off their decay channels during the hadronic cascade.

Let us first check how early $\phi$ mesons decouple from the rest of the system. Figure 6 shows the normalized distribution of freeze-out times for pions, protons, and $\phi$ mesons near midrapidity $|y|<1$ in central collisions $(\langle b\rangle=2.0 \mathrm{fm})$. Clearly, $\phi$ mesons decouple earlier than pions and protons. The freeze-out time distribution for $\phi$ mesons has a prominent peak at $\tau=8 \mathrm{fm} / c$, roughly equal to the time of completion of QGP hadronization in hydrodynamic simulations. This indicates that only very few rescatterings happen for $\phi$ mesons during the hadronic evolution. Similar results were obtained with the RQMD cascade in Ref. [57] for $\Omega$ baryons at SPS energies and in Ref. [58] for $\phi$ mesons and $\Omega$ baryons at RHIC energies. The freeze-out time distributions for pions and protons are broadened by both elastic scatterings and resonance decays. The long resonance decay tails of the distributions are important for interpreting the pion source function that was recently reconstructed by the PHENIX Collaboration [59] using imaging methods.

In Fig. 7, $p_{T}$ spectra for $\phi$ mesons from the hybrid model are compared with PHENIX [60] and STAR [61] data. Similar to the spectra for pions, kaons, and protons in Fig. 3, we see good agreement with experiment at low $p_{T}\left(p_{T}<\right.$ $1.5 \mathrm{GeV} / c)$. The discrepancy between our results and experiment at larger $p_{T}$ may indicate the appearance of a quark-antiquark recombination component in the intermediate $p_{T}$ region $[62,63]$. In the presence of such a component 


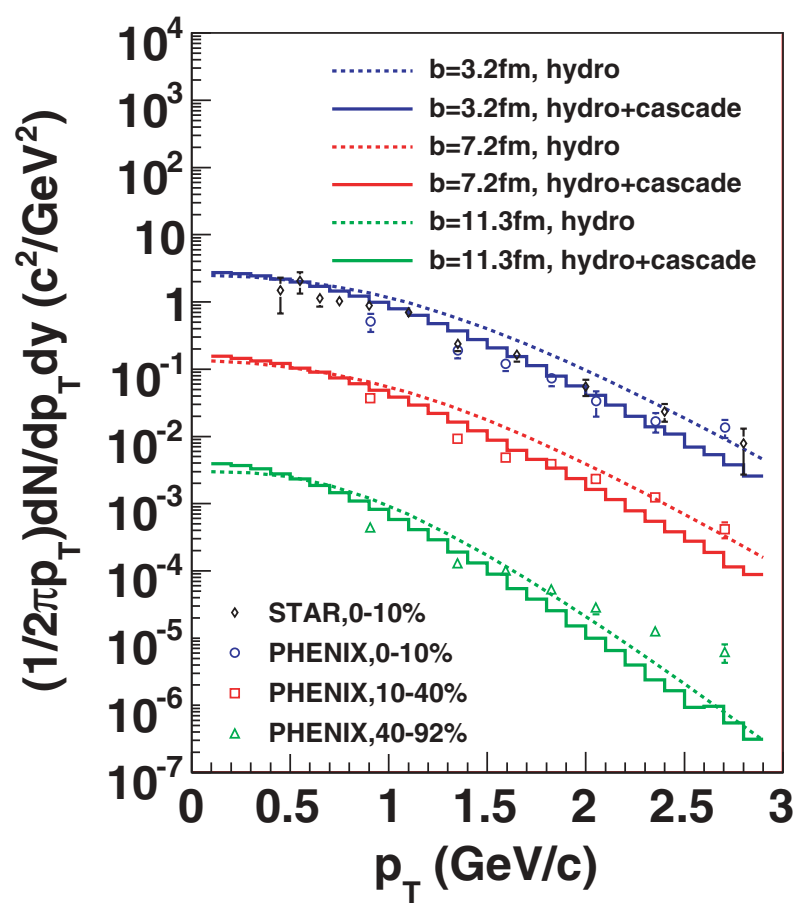

FIG. 7. (Color online) Transverse-momentum spectra for $\phi$ mesons reconstructed from $K^{+} K^{-}$decays in central (blue line), semicentral (red line), and peripheral (green line) $\mathrm{Au}+\mathrm{Au}$ collisions, compared with PHENIX [60] and STAR [61] data. Results from semicentral and peripheral collisions are divided by 10 and 100 , respectively. Predictions from ideal hydrodynamics with $T_{\text {th }}=$ $100 \mathrm{MeV}$ are also shown as dashed lines.

it is questionable to use the $\phi$-meson spectra over the whole available $p_{T}$ region to extract the thermal freeze-out temperature and flow for $\phi$ mesons [64]; such a thermal model fit [65] should be restricted to the region $p_{T}<1.5 \mathrm{GeV} / c$ even if data in that region are hard to obtain.

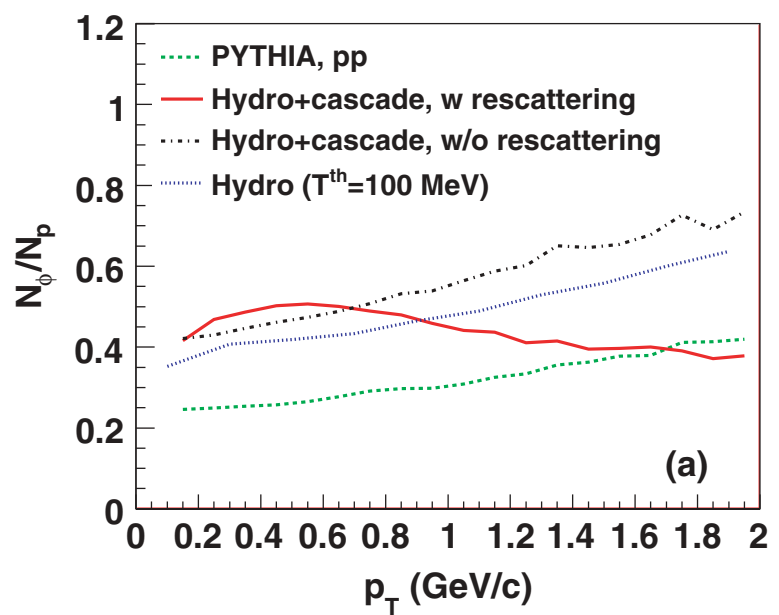

In the hydrodynamic model simulations with $T_{\text {th }}=$ $100 \mathrm{MeV}$, shown as dashed lines in Fig. 7, the $\phi$ mesons pick up more additional radial flow during the hadronic stage, resulting in flatter $p_{T}$ spectra than in the hybrid model and in the data in the low- $p_{T}$ region. As we will show further below, better $\phi$ data at lower $p_{T}$ and a simultaneous analysis of the differential elliptic flow in this region should allow to further discriminate between different descriptions of the hadronic rescattering stage.

The effects of radial flow, and the difference in how additional radial flow generated during the hadronic rescattering stage is picked up by protons and $\phi$ mesons (which have rather similar masses), can be enhanced by studying the $p_{T}$ or transverse kinetic energy dependence of the $\phi / p$ ratio. A thermalized medium without radial flow features $m_{T}$ scaling, i.e., all $m_{T}$ spectra have identical slopes, and for such a static fireball the $\phi / p$ ratio, when plotted as a function of transverse kinetic energy $\mathrm{KE}_{T} \equiv m_{T}-m_{0}$, would be a constant horizontal line. For a thermalized expanding medium, $m_{T}$ scaling is broken by radial flow (which couples differently to particles with different masses), resulting in a nonzero slope of the ratio $\phi / p\left(\mathrm{KE}_{T}\right)$. Perhaps somewhat counterintuitively, this slope of the $\phi / p$ ratio does not grow monotonically with the radial flow $v_{\perp}$ but, after an initial rise, decreases again when the flow becomes so large that the hadron $m_{T}$ spectra become very flat; in the limit of "infinite flow" (i.e., $\gamma_{\perp}=1 / \sqrt{1-v_{\perp}^{2}} \rightarrow \infty$ ) the hadron $m_{T}$ spectra, and thus their ratios, become again perfectly flat.

In Fig. 8 we show the $\phi / p$ ratio, both as a function of transverse kinetic energy (right panel) and of $p_{T}$ (left panel). It should be noted here that weak decay contribution is not included in proton yields. In the latter case the connection to radial flow is less straightforward, because the kinematics of the transformation from $m_{T}$ to $p_{T}$ depends on mass and introduces additional growth with $p_{T}$ for the ratio. In both representations one sees, however, by comparing the curves for the hydro+cascade model without rescattering (corresponding

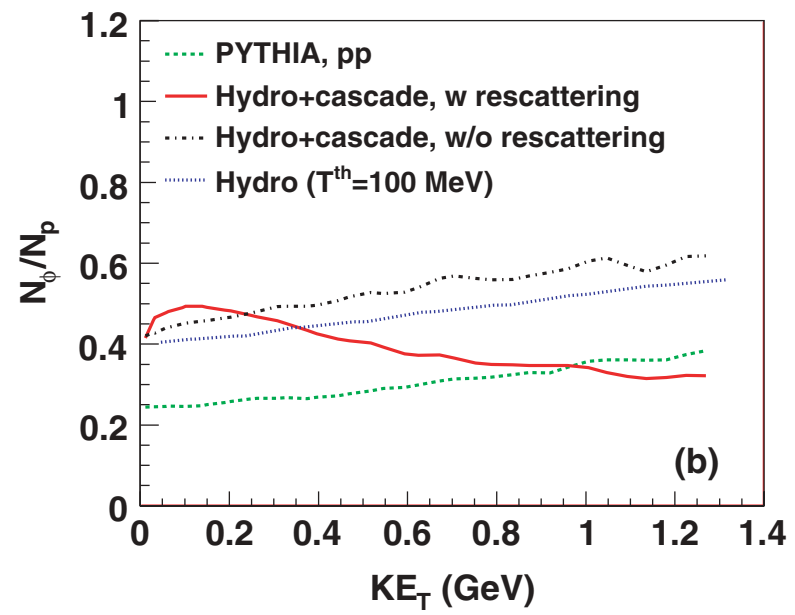

FIG. 8. (Color online) The $\phi / p$ ratio as a function of $p_{T}$ (left panel) and of transverse kinetic energy $\mathrm{KE}_{T} \equiv m_{T}-m_{0}$ (right panel) for different scenarios: central $\mathrm{Au}+\mathrm{Au}$ collisions in the hybrid model, without hadronic rescattering, and in the hydrodynamic model with $T_{\mathrm{th}}=$ $100 \mathrm{MeV}$ (dotted). The corresponding ratio for proton-proton collisions (extracted from the PYTHIA fit to the experimental data shown in Fig. 10 below) is shown for comparison as the dashed line. See text for more discussion. 

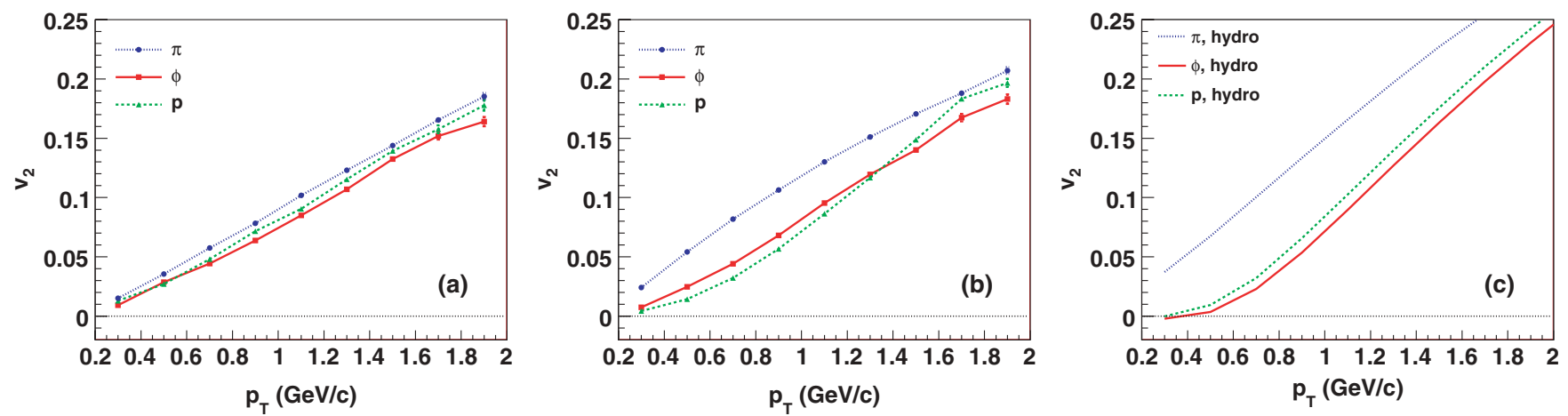

FIG. 9. (Color online) Transverse-momentum dependence of the elliptic flow parameters for pions (dotted blue), protons (dashed green), and $\phi$ mesons (solid red), for Au+Au collisions at $b=7.2 \mathrm{fm}$. (a) Before hadronic rescattering. (b) After hadronic rescattering. (c) Ideal hydrodynamics with $T_{\mathrm{th}}=100 \mathrm{MeV}$. The results for pions and protons are the same as shown in Fig. 5 .

to ideal hydrodynamics with $T_{\text {th }}=169 \mathrm{MeV}$ ) and for the ideal hydrodynamic model with $T_{\text {th }}=100 \mathrm{MeV}$, that (i) the ratio increases with $p_{T}$ or $\mathrm{KE}_{T}$ due to radial flow effects and that (ii) the rate of increase drops when the freeze-out temperature $T_{\text {th }}$ is decreased, due to buildup of additional radial flow. Surprisingly, the ratio increases even in $p p$ collisions, but for entirely different reasons, unrelated to collective flow: the $\phi$ spectrum from $p p$ collisions shown in Fig. 10 below is considerably flatter than the proton spectrum, leading to the prominent rise of the $\phi / p$ ratio with $p_{T}$. The most interesting feature of Fig. 8 is that the $\phi / p$ ratio from the hybrid model does not at all increase with $p_{T}$ or $\mathrm{KE}_{T}$ (except at very

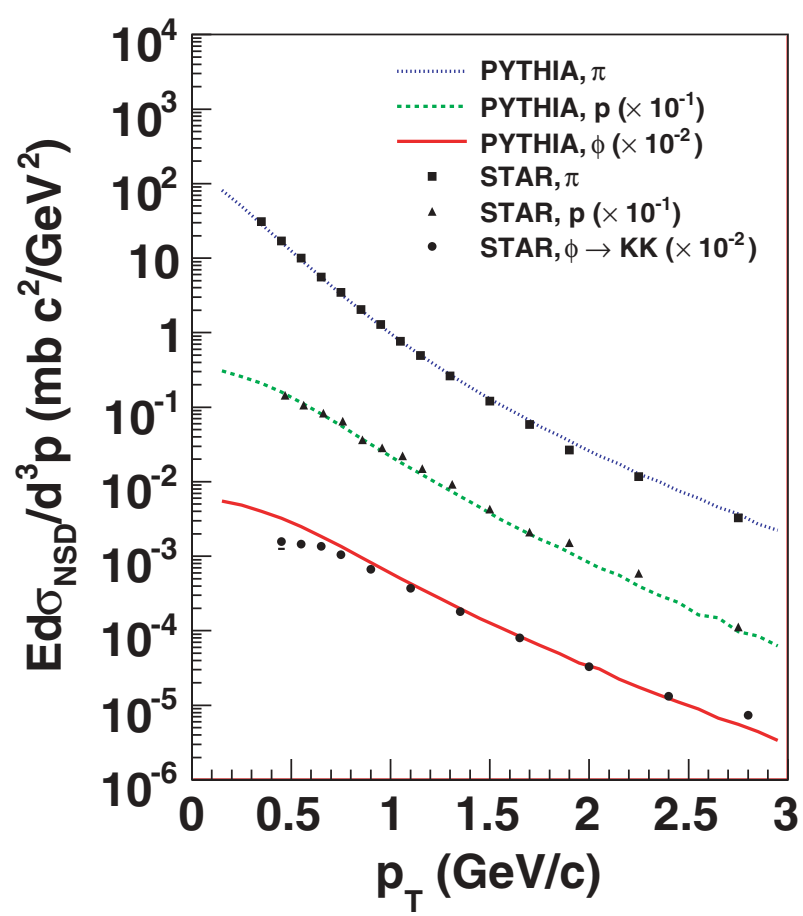

FIG. 10. (Color online) Invariant cross sections as a function of $p_{T}$ in nonsingly diffractive $p p$ collisions for pions, protons, [72] and $\phi$ mesons. Dotted, dashed, and solid lines are results from PYTHIA for pions, protons, and $\phi$ mesons, respectively. low $p_{T}<500 \mathrm{MeV} / c$ ). Instead, it decreases over almost the entire range of transverse kinetic energy shown in the figure. This decrease is due to the flattening of the proton spectrum by hadronically generated radial flow in which the weakly coupled $\phi$ mesons do not participate. The comparison with $p p$ collisions and hydrodynamic model simulations in Fig. 8 shows that the observation of such a decreasing $\phi / p$ ratio would be an unambiguous signature for early decoupling of $\phi$ mesons from the hadronic rescattering dynamics.

We now proceed to the discussion of dissipative effects during the hadronic rescattering stage on the differential elliptic flow $v_{2}\left(p_{T}\right)$. Figure 9 shows $v_{2}\left(p_{T}\right)$ from the hybrid model for $\pi, p$, and $\phi$. We consider semicentral collisions (20-30\% centrality), choosing impact parameter $b=7.2 \mathrm{fm}$. In the absence of hadronic rescattering we observe the hydrodynamically expected mass ordering $v_{2}^{\pi}\left(p_{T}\right)>v_{2}^{p}\left(p_{T}\right)>$ $v_{2}^{\phi}\left(p_{T}\right)$ [Fig. 9(a)], but just as in Fig. 5 (dashed lines) the mass splitting is small. Figure 9(b) shows the effects of hadronic rescattering: whereas the $v_{2}\left(p_{T}\right)$ curves for pions and protons separate as discussed before (at low $p_{T}$ the pion curve moves up while the proton curve moves down), $v_{2}\left(p_{T}\right)$ for the $\phi$ meson remains almost unchanged [66]. As a result of rescattering the proton elliptic flow ends up being smaller than that of the $\phi$ meson, $v_{2}^{p}\left(p_{T}\right)<v_{2}^{\phi}\left(p_{T}\right)$ for $0<p_{T}<$ $1.2 \mathrm{GeV} / c$, even though $m_{\phi}>m_{p}$. Hadronic dissipative effects are seen to be particle specific, depending on their scattering cross sections that couple them to the medium. The large cross section difference between the protons and $\phi$ mesons in the hadronic rescattering phase leads to a violation of the hydrodynamic mass ordering at low $p_{T}$ in the final state.

This is the most important new result of our work. Current experimental data $[67,68]$ neither confirm nor contradict this predicted behavior, due to the difficulty of reconstructing low- $p_{T} \phi$ mesons from their decay products. If it turns out that high-precision $\phi$-meson $v_{2}$ data at low $p_{T}$ show violation of mass ordering, it will be evidence for strong momentum anisotropy having developed already during the QGP stage, with the contribution carried by $\phi$ mesons not being redistributed in $p_{T}$ by late hadronic rescattering. At intermediate $p_{T}$, recent data $[67,68]$ confirm the prediction from the quark coalescence model $[69,70]$ that there the elliptic 

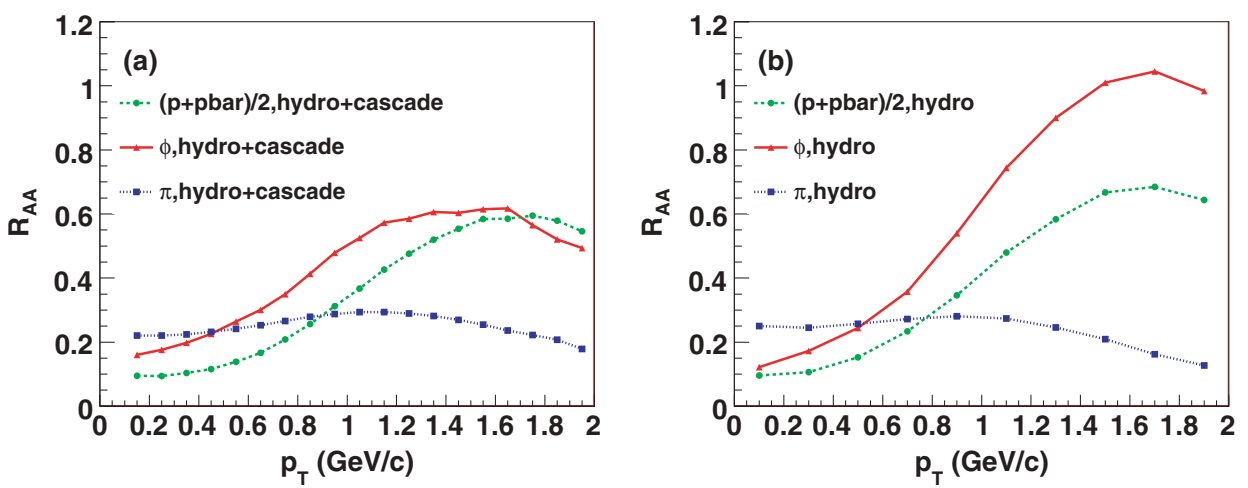

FIG. 11. (Color online) Nuclear modification factors $R_{A A}$ for pions (blue), $\phi$ mesons (red), and protons/antiprotons (green) for $\mathrm{Au}+\mathrm{Au}$ collisions at $b=3.2 \mathrm{fm}$ (corresponding to $0-10 \%$ centrality). Shown are predictions from (a) the hybrid model and (b) from ideal hydrodynamics with $T_{\text {th }}=100 \mathrm{MeV}$. flow should scale with the number of constituent quarks: $v_{2}^{\phi}\left(p_{T}\right) \approx v_{2}^{\pi, K}\left(p_{T}\right) \approx \frac{2}{3} v_{2}^{p}\left(p_{T}\right)$, in spite of the similar $\phi$ and $p$ masses that are much larger than those of the pions and $K$ mesons. We hope that the present article motivates an effort to extend these data to lower $p_{T}$ to test our prediction here that, at low $p_{T}, v_{2}^{p}\left(p_{T}\right)<v_{2}^{\phi}\left(p_{T}\right)$ in spite of $m_{\phi}>m_{p}$. Although the former observation suggests that at intermediate $p_{T}\left(2 \mathrm{GeV} / c<p_{T}<6 \mathrm{GeV} / c\right)$ quark coalescence during the quark-hadron phase transition controls the finally observed elliptic flow of all hadrons, without measurable distortion by subsequent hadronic reinteractions, confirmation of our prediction would confirm the importance of hadronic rescattering on low- $p_{T}$ hadrons, with results that depend on the magnitude of the scattering cross sections of the various hadron species.

We close this section with a discussion of the implications of our hybrid model results for the nuclear modification factor

$$
R_{A A}\left(p_{T}\right)=\frac{\frac{d N_{A A}}{p_{T} d p_{T} d y}}{N_{\text {coll }} \frac{d N_{p p}}{p_{T} d p_{T} d y}}=\frac{\frac{d N_{A A}}{p_{T} d p_{T} d y}}{T_{A A} \frac{d \sigma_{p p}}{p_{T} d p_{T} d y}} .
$$

The observed suppression of pion yields at intermediate to high $p_{T}$ [1] provides evidence of jet quenching in relativistic heavy-ion collisions. For baryons, this suppression effect is counteracted in the intermediate $p_{T}$ region by collective flow effects that, at low $p_{T}$, lead to a rise of the $p / \pi$ (or, more generally, heavy/light) ratio as a function of $p_{T}$. Collective flow effects extend into the intermediate $p_{T}$ region $2 \mathrm{GeV} / c<p_{T}<6 \mathrm{GeV} / c$ even though the hydrodynamic picture is known to gradually break down above $p_{T}>1.5-$ $2.5 \mathrm{GeV} / c$ [71]. Quark coalescence is one of the key mechanisms by which low- $p_{T}$ collectivity on the quark-gluon level is transferred to the hadron spectra at intermediate $p_{T}$ during the hadronization process $[62,63,69]$, leading to (unsuppressed) values of $R_{A A}$ (or of $R_{\mathrm{CP}}$, the ratio of yields per number of binary collisions in central and peripheral collisions) of order unity for baryons at $p_{T} \sim 2-3 \mathrm{GeV} / c[1,68]$. We will show that hadronic rescattering following QGP hadronization affects $R_{A A}$ at low $p_{T}$ instead.

The PHENIX [60] and STAR [68] Collaborations have recently measured $R_{\mathrm{CP}}$ for $\phi$ mesons. The PHENIX data show a suppression of $\phi$ mesons by about a factor 2 (with relatively large error bars) in the region $1 \mathrm{GeV} / c<p_{T}<3 \mathrm{GeV} / c$, consistent with that of pions, whereas protons and antiprotons are unsuppressed [60]. This seems to be in contradiction with collective flow arguments that predict $R_{\mathrm{CP}}^{\phi}>R_{\mathrm{CP}}^{p}$ because $m_{\phi}>m_{p}$ but consistent with the valence quark scaling predicted by the quark coalescence model $\{[62,63,69]$. The more recent and precise STAR data [68], however, show an $R_{\mathrm{CP}}$ for $\phi$ mesons that follows the one for pions and exceeds the one for protons for $p_{T}<1 \mathrm{GeV} / c$ but then follows the rise of the proton $R_{\mathrm{CP}}$ above the pion one for $p_{T}>1 \mathrm{GeV} / c$, lagging only slightly behind the protons and reaching a value halfway between pions and protons in the region $p_{T} \sim 2-$ $3 \mathrm{GeV} / c$, where $R_{\text {CP }}^{p}$ peaks at a value of $\sim 1$.

Given this somewhat contradictory experimental situation, we offer a prediction from our hybrid model (cautioning beforehand that this model does not include any quarkrecombination contributions that are expected to become important above $p_{T} \gtrsim 1.5-2 \mathrm{GeV} / c$ ) in Fig. 11 . To construct this figure, we first fitted the experimentally measured $p_{T}$ spectra for pions and protons [72] as well as for $\phi$ mesons [73] in nonsingly diffractive (NSD) $p p$ collisions (i.e., inelastic collisions excluding single diffractive events). The fit, shown in Fig. 10, is performed with the help of the event generator PYTHIA 6.403 [74] that, once properly tuned, yields smooth reference $p_{T}$-spectra for $p p$ collisions. PYTHIA is based on leading-order perturbative QCD for semihard processes combined with a Lund string fragmentation scheme for soft particles. It works quite well for pions, protons, and $\phi$ mesons with default parameters [74], except for a necessary readjustment of the $K$ factor to $K=1.8$. We note that here exceptionally this comparison includes all resonance decays, including weak ones, because the STAR data show the inclusive spectra. We take the resulting spectra as our $p p$ reference, after removing weak decay contributions and multiplying them with the ratio $\sigma_{\text {in }} / \sigma_{\mathrm{NSD}}$ to correct for the NSD trigger. For the required cross sections PYTHIA provides the estimates $\sigma_{\mathrm{NSD}}=32 \mathrm{mb}$ and $\sigma_{\mathrm{in}}=42 \mathrm{mb}$.

With these reference spectra the nuclear modification factors $R_{A A}$ can now be calculated from the results shown in Figs. 1, 3, and 7. For pions, protons, and $\phi$ mesons they are shown as functions of $p_{T}$ in Fig. 11, for $\mathrm{Au}+\mathrm{Au}$ collisions at impact parameter $b=3.2 \mathrm{fm}$ (i.e. $0-10 \%$ centrality). Figure 11(a) shows the predictions for the hybrid model. Although for pions $R_{A A}\left(p_{T}\right)$ is almost flat, $R_{A A}^{\pi} \sim 0.15-0.25$, the $R_{A A}\left(p_{T}\right)$ curves for protons and $\phi$ mesons increase with $p_{T}$ as expected from radial flow arguments (radial flow hardens the $p_{T}$ spectra for heavy particles). The rate of increase for the $\phi$ mesons is very similar to that for protons, culminating in a peak value of $\sim 60 \%$ at $p_{T} \sim 1.2-1.4 \mathrm{GeV} / c$ for $\phi$ 's, 
whereas the $R_{A A}$ for protons peaks at a value of $\sim 60 \%$ near $p_{T} \sim 1.8 \mathrm{GeV} / c$. Figure 11 (b) shows the corresponding curves for the ideal fluid dynamical simulation with $T_{\text {th }}=100 \mathrm{MeV}$. For pions and protons, the differences to the hybrid model are minor (at least in the $p_{T}$ range covered in the figure), reiterating the observation made in connection with Fig. 1 that the buildup of additional radial flow during the hadronic stage is similar in both models and viscous effects become clearly recognizable only at larger $p_{T}$. For $\phi$ mesons one observes a much faster rise of $R_{A A}\left(p_{T}\right)$ in the hydrodynamic approach, resulting in a larger peak value of $\sim 105 \%$ at a larger $p_{T}$ value $(\sim 1.7 \mathrm{GeV} / c)$ than for the hybrid model. The reason for these effects is obviously the larger amount of radial flow picked up during the hadronic stage in the hydrodynamic model and the resulting hardening of the $\phi$ spectrum. The much weaker rise of $R_{A A}^{\phi}\left(p_{T}\right)$ in the hybrid model can thus be traced directly to the lack of $\phi$ meson rescattering during the hadronic stage.

We note that, even in the hydrodynamic model, the nuclear modification factor $R_{A A}\left(p_{T}\right)$ does not show a monotonic mass ordering at low $p_{T}$. Naive expectations based on the mass ordering of the spectral slopes (that reflect radial flow effects) are invalidated by the fact that the $\phi p_{T}$ spectra from $p p$ collisions are flatter than the corresponding proton spectra. Because these spectra enter the denominator of $R_{A A}$, they distort its $p_{T}$ dependence differently for protons and $\phi$ mesons.

We also comment that at $p_{T} \sim 2 \mathrm{GeV} / c$, the characteristics of the observed mass-scaling violation in Fig. 11(a) are qualitatively similar to those expected (and observed) in the quark coalescence picture at intermediate $p_{T}(2 \mathrm{GeV} / c<$ $p_{T}<6 \mathrm{GeV} / c$ ) [70]. The differences are quantitative: our prediction for $R_{A A}$ features neither a monotonic mass ordering at low $p_{T}$ nor the strict valence quark scaling predicted by the quark-coalescence picture at intermediate $p_{T}$.

\section{CONCLUSIONS}

We have studied effects of hadronic dissipation on the spectra, differential elliptic flow, and nuclear modification factor of pions, kaons, protons, and $\phi$ mesons from $\mathrm{Au}+\mathrm{Au}$ collisions at RHIC, using a hybrid model that treats the early QGP phase macroscopically as a perfect fluid and the late hadronic phase microscopically with a hadronic cascade. For transverse momenta below $1.5 \mathrm{GeV} / c$ and not too peripheral collisions, the hybrid model gives a reasonable description of the measured pion, kaon, proton, and $\phi$ meson $p_{T}$ spectra. In peripheral collisions ( $b=9 \mathrm{fm}$ and larger) the model spectra tend to be somewhat steeper than measured. The centrality dependence of the differential elliptic flow $v_{2}\left(p_{T}\right)$ of pions, kaons, and protons is better described by the hybrid model than in a purely hydrodynamic approach.

For pions, kaons, and protons, which have relatively large scattering cross sections, hadronic rescattering is seen to generate additional collective transverse flow but not so for the much more weakly interacting $\phi$ mesons. However, even for pions and protons the extra hadronic transverse flow effects are not "ideal" but exhibit obvious viscous features: Their $p_{T}$ spectra are hardened, whereas the growth of their elliptic flow $v_{2}\left(p_{T}\right)$ with increasing $p_{T}$ is tempered by viscous corrections whose importance is in both cases observed to increase with transverse momentum. The well-known mass splitting of the differential elliptic flow $v_{2}\left(p_{T}\right)$ observed in hydrodynamic models is seen to be mostly generated during the hadronic rescattering phase and to be largely due to a redistribution of the momentum anisotropy built up during the QGP stage. This redistribution is caused by the mass-dependent flattening of the transverse-momentum spectra by additional radial flow generated during the hadronic stage. The much more weakly interacting $\phi$ mesons do not participate in this additional radial flow and thus are not affected by this redistribution of momentum anisotropies: their differential elliptic flow remains almost unaffected by hadronic rescattering. The net result of dissipative hadronic rescattering is therefore that the differential elliptic flow $v_{2}\left(p_{T}\right)$ of protons drops below that of the $\phi$ mesons, in violation of the hydrodynamic mass ordering. A similar violation of the mass ordering is seen in the nuclear modification factor $R_{A A}\left(p_{T}\right)$ at $p_{T} \sim 2 \mathrm{GeV} / c$, where, after hadronic rescattering, the curve for $\phi$ mesons ends up between those for pions and protons even though the $\phi$ is heavier than both of them. For the $\phi / p$ ratio, the lack of interaction between the $\phi$ mesons and its accelerating hadronic environment should manifest itself in an unexpected but unambiguous decrease with increasing transverse kinetic energy.

The results presented here underscore the conclusion of Ref. [15] that hadronic dissipation may be very important at RHIC and at lower beam energies and should be properly accounted for in attempts to quantitatively account for the experimental data collected from heavy-ion collisions. With $v_{2}\left(p_{T}\right)$ and $R_{A A}\left(p_{T}\right)$ for low- $p_{T} \phi$ mesons and the dependence of the $\phi / p$ ratio on $p_{T}$ or transverse kinetic energy $\mathrm{KE}_{T}$, we have identified three additional critical observables that should be helpful in sorting out the interplay between hydrodynamic evolution during the early QGP stage and dissipative hadronic expansion during the late stage of the hot and dense fireballs created in these collisions. An accurate extraction of the value for the specific shear viscosity $\eta / s$ of the QGP created at RHIC requires a proper accounting for effects from late hadronic viscosity. Here, an attempt has been made to do this, by coupling the hydrodynamic model to a hadronic cascade.

\section{ACKNOWLEDGMENTS}

This work was supported by the US DOE under contracts DE-FG02-01ER41190 (U.H.), DE-AC02-98CH10886 (D.K.), and DE-FG02-87ER40331.A008 (R.L.). The work of T.H. was partly supported by Grant-in-Aid for Scientific Research no. 19740130.
[1] The experimental situation is summarized in I. Arsene et al. (BRAHMS Collaboration), Nucl. Phys. A757, 1 (2005); B. B. Back et al. (PHOBOS Collaboration), ibid. A757, 28 (2005);
J. Adams et al. (STAR Collaboration), ibid. A757, 102 (2005); K. Adcox et al. (PHENIX Collaboration), ibid. A757, 184 (2005). 
[2] For theoretical reviews see P. Huovinen, in Quark-Gluon Plasma 3, edited by R. C. Hwa and X.-N. Wang (World Scientific, Singapore, 2004), p. 600; P. F. Kolb and U. Heinz, ibid., p. 634; U. Heinz, in Extreme $Q C D$, edited by G. Aarts and S. Hands (University of Wales, Swansea, 2006), p. 3.

[3] T. Hirano and M. Gyulassy, Nucl. Phys. A769, 71 (2006).

[4] P. Romatschke and U. Romatschke, Phys. Rev. Lett. 99, 172301 (2007).

[5] H. Song and U. Heinz, Phys. Lett. B658, 279 (2008).

[6] G. Policastro, D. T. Son, and A. O. Starinets, Phys. Rev. Lett. 87, 081601 (2001); P. K. Kovtun, D. T. Son, and A. O. Starinets, Phys. Rev. Lett. 94, 111601 (2005).

[7] J. Y. Ollitrault, Phys. Rev. D 46, 229 (1992).

[8] C. Adler et al. (STAR Collaboration), Phys. Rev. Lett. 87, 182301 (2001); J. Adams et al. (STAR Collaboration), ibid. 92, 052302 (2001).

[9] K. Adcox et al. (PHENIX Collaboration), Phys. Rev. Lett. 89, 212301 (2002); S. S. Adler et al. (PHENIX Collaboration), ibid. 91, 182301 (2003).

[10] P. Huovinen, P. F. Kolb, U. Heinz, P. V. Ruuskanen, and S. A. Voloshin, Phys. Lett. B503, 58 (2001).

[11] C. Adler et al. (STAR Collaboration), Phys. Rev. C 66, 034904 (2002); C. Alt et al. (NA49 Collaboration), ibid. 68, 034903 (2003).

[12] B. B. Back et al. (PHOBOS Collaboration), Phys. Rev. Lett. 89, 222301 (2002).

[13] T. Hirano, Phys. Rev. C 65, 011901 (2001).

[14] T. Hirano and K. Tsuda, Phys. Rev. C 66, 054905 (2002).

[15] T. Hirano, U. Heinz, D. Kharzeev, R. Lacey, and Y. Nara, Phys. Lett. B636, 299 (2006); J. Phys. G 34, S879 (2007).

[16] A. Muronga, Phys. Rev. Lett. 88, 062302 (2002) [Erratum: ibid. 89, 159901 (2002)]; Phys. Rev. C 69, 034903 (2004); 76, 014909 and 014910 (2007); A. Muronga and D. H. Rischke, arXiv:nuclth/0407114; D. A. Teaney, J. Phys. G: Nucl. Part. Phys. 30, S1247 (2004); R. Baier, P. Romatschke, and U. A. Wiedemann, Phys. Rev. C 73, 064903 (2006); A. K. Chaudhuri and U. Heinz, J. Phys.: Conf. Ser. 50, 251 (2006); R. Baier and P. Romatschke, Eur. Phys. J. C 51, 677 (2007); P. Romatschke, ibid. 52, 203209 (2007); U. Heinz, H. Song, and A. K. Chaudhuri, Phys. Rev. C 73, 034904 (2006); A. K. Chaudhuri, ibid. 74, 044904 (2006); arXiv:0704.0134 [nucl-th]; and arXiv:0708.1252 [nucl-th].

[17] A. Adil, H. J. Drescher, A. Dumitru, A. Hayashigaki, and Y. Nara, Phys. Rev. C 74, 044905 (2006).

[18] T. Lappi and R. Venugopalan, Phys. Rev. C 74, 054905 (2006).

[19] H. J. Drescher and Y. Nara, Phys. Rev. C 75, 034905 (2007).

[20] F. Cooper and G. Frye, Phys. Rev. D 10, 186 (1974).

[21] A. Dumitru, S. A. Bass, M. Bleicher, H. Stöcker, and W. Greiner, Phys. Lett. B460, 411 (1999); S. A. Bass, A. Dumitru, M. Bleicher, L. Bravina, E. Zabrodin, H. Stöcker, and W. Greiner, Phys. Rev. C 60, 021902(R) (1999); S. A. Bass and A. Dumitru, ibid. 61, 064909 (2000).

[22] D. Teaney, J. Lauret, and E. V. Shuryak, Phys. Rev. Lett. 86, 4783 (2001).

[23] C. Nonaka and S. A. Bass, Nucl. Phys. A774, 873 (2006); Phys. Rev. C 75, 014902 (2007).

[24] Note that the authors of Ref. [23] use different algorithms for both the hydrodynamic and hadronic cacade codes than employed here and in Ref. [15].

[25] P. Colella and P. R. Woodward, J. Comput. Phys. 54, 174 (1984).

[26] T. Hirano, Phys. Rev. Lett. 86, 2754 (2001); T. Hirano, K. Tsuda, and K. Kajimoto, nucl-th/0011087.
[27] V. Schneider, U. Katscher, D. H. Rischke, B. Waldhauser, J. A. Maruhn, and C.-D. Munz, J. Comput. Phys. 105, 92 (1993); D. H. Rischke, S. Bernard, and J. A. Maruhn, Nucl. Phys. A595, 346 (1995); D. H. Rischke, Y. Pürsün, and J. A. Maruhn, ibid. A595, 383 (1995).

[28] P. Braun-Munzinger, D. Magestro, K. Redlich, and J. Stachel, Phys. Lett. B518, 41 (2001).

[29] H. Bebie, P. Gerber, J. L. Goity, and H. Leutwyler, Nucl. Phys. B378, 95 (1992).

[30] N. Arbex, F. Grassi, Y. Hama, and O. Socolowski Jr., Phys. Rev. C 64, 064906 (2001).

[31] D. Teaney, nucl-th/0204023.

[32] P. F. Kolb and R. Rapp, Phys. Rev. C 67, 044903 (2003).

[33] P. Huovinen, arXiv:0710.4379 [nucl-th].

[34] The main difference is in baryon-antibaryon annihilation channels where the hadronic cascade models lack a consistent description of inverse processes involving more than two colliding particles. This lack of detailed balance leads to an excess loss of baryons and antibaryons during the hadronic rescattering phase $[21,35]$.

[35] R. Rapp and E. V. Shuryak, Phys. Rev. Lett. 86, 2980 (2001); C. Greiner and S. Leupold, J. Phys. G: Nucl. Part. Phys. 27, L95 (2001).

[36] A. Adil and M. Gyulassy, Phys. Rev. C 72, 034907 (2005).

[37] B. B. Back et al. (PHOBOS Collaboration), Phys. Rev. Lett. 91, 052303 (2003).

[38] P. F. Kolb, U. Heinz, P. Huovinen, K. J. Eskola, and K. Tuominen, Nucl. Phys. A696, 197 (2001).

[39] B. B. Back et al. (PHOBOS Collaboration), Phys. Rev. C 65, 061901 (2002).

[40] A. J. Kuhlman and U. Heinz, Phys. Rev. C 72, 037901 (2005); [That paper uses a slightly smaller soft fraction $\alpha=0.75$.]

[41] D. Kharzeev and M. Nardi, Phys. Lett. B507, 121 (2001); D. Kharzeev and E. Levin, ibid. B523, 79 (2001); D. Kharzeev, E. Levin, and M. Nardi, Phys. Rev. C 71, 054903 (2005); Nucl. Phys. A730, 448 (2004).

[42] T. Hirano and Y. Nara, Nucl. Phys. A743, 305 (2004).

[43] A. Kuhlman, U. Heinz, and Y. V. Kovchegov, Phys. Lett. B638, 171 (2006).

[44] H. J. Drescher, A. Dumitru, C. Gombeaud, and J. Y. Ollitrault, Phys. Rev. C 76, 024905 (2007).

[45] Y. Nara, N. Otuka, A. Ohnishi, K. Niita, and S. Chiba, Phys. Rev. C 61, 024901 (1999).

[46] H. Sorge et al., Phys. Lett. B271, 37 (1991); H. Sorge, L. Winckelmann, H. Stöcker, and W. Greiner, Z. Phys. C 59, 85 (1993).

[47] S. A. Bass et al., Prog. Part. Nucl. Phys. 41, 225 (1998).

[48] S. S. Adler et al. (PHENIX Collaboration), Phys. Rev. C 69, 034909 (2004).

[49] M. Gyulassy and T. Matsui, Phys. Rev. D 29, 419 (1984).

[50] P. V. Ruuskanen, Phys. Lett. B147, 465 (1984).

[51] D. Teaney, Phys. Rev. C 68, 034913 (2003).

[52] J. Adams et al. (STAR Collaboration), Phys. Rev. C 72, 014904 (2005).

[53] M. Miller and R. Snellings, nucl-ex/0312008; X. Zhu, M. Bleicher, and H. Stoecker, Phys. Rev. C 72, 064911 (2005); S. Manly et al. (PHOBOS Collaboration), Nucl. Phys. A774, 523 (2006); R. Andrade, F. Grassi, Y. Hama, T. Kodama, and O. Socolowski Jr., Phys. Rev. Lett. 97, 202302 (2006); B. Alver et al. (PHOBOS Collaboration), Int. J. Mod. Phys. E, 
16, 3331 (2008); W. Broniowski, P. Bozek, and M. Rybczynski, Phys. Rev. C 76, 054905 (2007).

[54] P. F. Kolb, J. Sollfrank, and U. Heinz, Phys. Lett. B459, 667 (1999).

[55] P. F. Kolb, J. Sollfrank, and UHeinz, Phys. Rev. C 62, 054909 (2000).

[56] A. Shor, Phys. Rev. Lett. 54, 1122 (1985).

[57] H. van Hecke, H. Sorge, and N. Xu, Phys. Rev. Lett. 81, 5764 (1998).

[58] Y. Cheng, F. Liu, Z. Liu, K. Schweda, and N. Xu, Phys. Rev. C 68, 034910 (2003).

[59] S. S. Adler et al. (PHENIX Collaboration), Phys. Rev. Lett. 98, 132301 (2007).

[60] S. S. Adler et al. (PHENIX Collaboration), Phys. Rev. C 72, 014903 (2005).

[61] J. Adams et al. (STAR Collaboration), Phys. Lett. B612, 181 (2005).

[62] V. Greco, C. M. Ko, and P. Levai, Phys. Rev. Lett. 90, 202302 (2003); Phys. Rev. C 68, 034904 (2003).

[63] R. J. Fries, B. Muller, C. Nonaka, and S. A. Bass, Phys. Rev. Lett. 90, 202303 (2003); Phys. Rev. C 68, 044902 (2003).
[64] K. Schweda and N. Xu, Acta Phys. Hung. A 22, 103 (2005); K. Schweda AIP Conf. Proc. 828, 69 (2006).

[65] E. Schnedermann, J. Sollfrank, and U. Heinz, Phys. Rev. C 48, 2462 (1993).

[66] J. H. Chen et al., Phys. Rev. C 74, 064902 (2006).

[67] S. Afanasiev et al. (PHENIX Collaboration), Phys. Rev. Lett. 99, 052301 (2007).

[68] B. I. Abelev et al. (STAR Collaboration), Phys. Rev. Lett. 99, 112301 (2007).

[69] D. Molnar and S. A. Voloshin, Phys. Rev. Lett. 91, 092301 (2003).

[70] C. Nonaka, R. J. Fries, and S. A. Bass, Phys. Lett. B583, 73 (2004).

[71] U. Heinz, J. Phys. G: Nucl. Part. Phys. 31, S717 (2005).

[72] J. Adams et al. (STAR Collaboration), Phys. Lett. B616, 8 (2005).

[73] J. Adams et al. (STAR Collaboration), Phys. Lett. B612, 181 (2005).

[74] T. Sjostrand, S. Mrenna, and P. Skands, J. High Energy Phys. 05 (2006) 026. 